\title{
On Small Zeros of Dirichlet $L$-Functions
}

\author{
By Peter J. Weinberger
}

To D. H. Lehmer for his 70 th birthday

\begin{abstract}
A method is given for calculating the value of Dirichlet $L$-functions near the real axis in the critical strip. As an application, some zeros for zeta functions of complex quadratic fields are calculated.
\end{abstract}

1. Introduction. The Dirichlet $L$-functions $L(s, \chi)=\Sigma_{n=1}^{\infty} \chi(n) / n^{s}$ are commonly believed to have all their nontrivial zeros on the critical line $\operatorname{Re}(s)=1 / 2$, although no single $L$-function is known to have this property, nor are any counterexamples known. Extrapolation from the experience of [1] for $\zeta(s)$ indicates that one is unlikely to find counterexamples by calculating zeros up the critical line. The zeros near $s=1 / 2$ are of some independent interest. If $\chi$ is a quadratic character with $\chi(-1)=$ -1 , then zeros of $L(s, \chi)$ especially close to $s=1 / 2$ have an effect on the class numbers of complex quadratic fields [2]. $L$-functions for these characters with conductors no greater than 800000 are known not to have any real zeros in the critical strip [9], [10]. Also, zeros near $s=1 / 2$ for any $\chi$ clearly affect, through the functional equation, the argument of the Gauss sum, which is connected with a conjecture of Kummer.

In this paper I give a method for finding zeros of $L(s, \chi)$ with $\operatorname{Re}(s)=1 / 2$ and $\operatorname{Im}(s)$ fairly small, say $\operatorname{Im}(s)<1$. All known methods for finding zeros of these functions depend on the evaluation of some auxiliary function which has the same zeros. The values of the auxiliary function are the data which some scheme of inverse interpolation uses to locate the zeros of the $L$-function. The method used in this paper requires $O\left(k^{1 / 2} \log k\right)$ operations for each function evaluation, which compares favorably to the method of [3], [4] which requires more than $k$ steps. For the purposes of computation, therefore, the present work is a $k$-analogue of the paper of Lehmer $[5]$.

A rough guide to the contents of the sections is as follows. Section 2 contains results used in the calculation of some zeros of $L(s, \chi)$ for the type of quadratic characters mentioned above. Section 3 gives a completely independent check on the calculation. Section 4 presents the actual numbers, and Section 5 contains further observations on general $\chi$.

The methods in this paper were developed for application in [2] and so are, in considerable measure, joint work with Hugh Montgomery.

Received July 19, 1974.

AMS (MOS) subject classifications (1970). Primary 10H10, 10-04: Secondary 12A25, $12-04$. Copyrigit @ 1975, American Mathematical Society 
2. The Method. In this section $\chi$ denotes a primitive quadratic character defined modulo $k$. This approach to determining zeros of $L(s, \chi)$ starts with the functional equation in the form

$$
\begin{aligned}
\xi(s, \chi)= & \left(\frac{k}{\pi}\right)^{(s+a) / 2} \Gamma\left(\frac{s+a}{2}\right) L(s, \chi)=\left(\frac{k}{\pi}\right)^{(s+a) / 2} \sum_{n=1}^{\infty} \frac{\chi(n)}{n^{s}} \Gamma\left(\frac{s+a}{2}, \frac{\pi n^{2}}{k}\right) \\
& +\left(\frac{k}{\pi}\right)^{(1+a-s) / 2} \sum_{n=1}^{\infty} \frac{\chi(n)}{n^{1-s}} \Gamma\left(\frac{1+a-s}{2}, \frac{\pi n^{2}}{k}\right) .
\end{aligned}
$$

Here $\Gamma(w, \alpha)=\int_{\alpha}^{\infty} x^{w-1} e^{-x} d x$, and $a=(1-\chi(-1)) / 2$. This is the functional equation since the right-hand side is invariant if $s$ is changed to $1-s$. It will be seen that this formula is most satisfactory when $\operatorname{Im}(s)$ is near zero. I shall not prove this formula since it is actually an intermediate step in the usual proof of the functional equation which uses theta functions. If $s=1 / 2+i t$, then the function $Z(t, \chi)=$ $\xi(1 / 2+i t, \chi)$ is real, and whenever $Z(t, \chi)$ changes sign, $L(1 / 2+i t, \chi)$ has a zero. To use (1) to calculate $Z(t, \chi)$ it is necessary to bound the error made in truncating the series. I shall assume that $\chi(-1)=-1$ throughout the rest of this section, since this is the only case for which calculations were actually performed. This restriction only affects Lemma 1.

First note that $\operatorname{Re}(w) \leqslant 1$ implies that

$$
|\Gamma(w, \alpha)|<e^{-\alpha} x^{\operatorname{Re}(w)-1} .
$$

Hence, writing $s=\sigma+i t, \quad \sigma \leqslant 1$,

$$
\begin{aligned}
\mid\left(\frac{k}{\pi}\right)^{(\sigma+1) / 2} \sum_{N+1}^{\infty} \frac{\chi(n)}{n^{s}} & \Gamma\left(\frac{s+1}{2}, \frac{\pi n^{2}}{k}\right) \mid<\frac{k}{\pi} \sum_{N+1}^{\infty} \frac{e^{-\pi n^{2} / k}}{n} \\
& <\frac{k}{\pi} \int_{N}^{\infty} \frac{e^{-\pi x^{2} / k} d x}{x} \leqslant \frac{k^{2}}{2 \pi^{2} N^{2}} e^{-\pi N^{2} / k} .
\end{aligned}
$$

This bound is independent of $s$, and also proves the following lemma.

LEMMA 1. If $s=1 / 2+i t$, then

$$
\left|Z(t, \chi)-2 \operatorname{Re}\left[\sum_{n=1}^{N}\left(\frac{k}{\pi}\right)^{(s+1) / 2} \frac{\chi(n)}{n^{s}} \Gamma\left(\frac{s+1}{2}, \frac{\pi n^{2}}{k}\right)\right]\right|<\frac{k^{2} e^{-N^{2} \pi / k}}{\pi^{2} N^{2}} .
$$

The first term of the series is, for large $k$ and small $|t|$, close to $(k / \pi)^{3 / 4} \Gamma(3 / 4)$, so when $N$ is larger than $(k \log k)^{1 / 2}$ the truncation error for the series becomes very small. In the calculations described below I took $N=(28 k / \pi)^{1 / 2}$ which makes the truncation error no larger than $1.57 \cdot 10^{-9}$ for $k$ less than 200000 .

The principal difficulty in using the formula in Lemma 1 to calculate $Z(t, \chi)$ is calculating the values of $\Gamma(3 / 4+i t / 2, \alpha)$. To see what is involved, consider $k=115147$. 
Here $N=1013$, so $\alpha$ varies over the interval $\left(2.7 \cdot 10^{-5}, 28.0\right)$. Hence the evaluation of $Z(t, \chi)$ requires about 1000 calculations of $\Gamma(3 / 4+i t / 2, \alpha)$ with the same value of $t$. For small $\alpha$ the value is clearly close to $\Gamma(3 / 4+i t / 2)$, so some sort of power series approximation seems reasonable. For large $\alpha$, the bound (2) indicates that the function can be sufficiently well approximated by Gauss-Laguerre integration using a few points. In between it seems reasonable to construct a table and interpolate in it, by some means, to the desired argument. One would like to calculate the integrals sufficiently accurately that the accumulated truncation and rounding error for the integrals is about as large as the bound for the truncation error in Lemma 1.

In this paper, the term truncation error refers to the error due to the use of approximations to the limiting processes of analysis, while rounding error refers to the error due to using finite-precision arithmetic.

LEmma 2. Suppose $N+2<\alpha \leqslant 0$ and $0 \leqslant \operatorname{Re}(w) \leqslant 1$. Then

$$
\left|\Gamma(w, \alpha)-\left(\Gamma(w)-\sum_{j=0}^{N} \frac{(-1)^{j} x^{j+s}}{(j+s) j !}\right)\right|<\frac{\alpha^{\mathrm{Re}(w)+N+1}}{|N+1+w|(N+1) !}\left(1-\frac{\alpha}{N+2}\right)^{-1} .
$$

Proof.

$$
\Gamma(w, \alpha)=\Gamma(w)-\int_{0}^{\alpha} x^{w-1} \sum_{j=0}^{\infty} \frac{(-1)^{j} x^{j}}{j !} d x=\Gamma(w)-\sum_{j=0}^{N} \frac{(-1)^{j} x^{j+s}}{(j+s) j !}+E_{N},
$$

where

$$
E_{N}<\frac{\alpha^{\mathrm{Re}(w)}}{|N+1+w|} \sum_{j=N+1}^{\infty} \frac{\alpha^{j}}{j !} \leqslant \frac{\alpha^{\mathrm{Re}(w)+N+1}}{|N+1+w|(N+1) !} \sum_{j=0}^{\infty}\left(\frac{\alpha}{N+2}\right)^{j} \text {. Q.E.D. }
$$

Some typical bounds for this truncation error for $\operatorname{Re}(w)=.75$ are $2 \cdot 10^{-15}$ for $\alpha=.5, N=12,910^{-16}$ for $\alpha=1.5, N=18$, and $9 \cdot 10^{-15}$ for $\alpha=3.2, N=24$. An alternative approach to calculating $\Gamma(w, \alpha)$ for small $\alpha$ is to integrate $\int_{0}^{\alpha} x^{w-1} e^{-x} d x$ by parts $N$ times, but this seems to lead to somewhat larger error terms. The value of $\Gamma(w)$ required for the application of Lemma 2 can easily be found by using the relation $w \Gamma(w)=\Gamma(w+1)$ to shift the argument to the right, and then using Stirling's approximation. My program calculates $\Gamma(w+8)$ using 6 terms of the asymptotic series, which gives an error of no more than $2 \cdot 10^{-14}$.

In order to justify the approximations used for larger $\alpha$, I need some unpleasant estimates.

Lemma 3. Suppose $w=u+i v$ with $0 \leqslant u \leqslant 1,0 \leqslant v$, and that $0<c<$ $a \leqslant x_{j} \leqslant b$, for $1 \leqslant j \leqslant n$. Let $C$ be a simple closed contour containing all the $x_{j}$ in its interior. Then

$$
\begin{gathered}
\left|\frac{1}{2 \pi i} \int_{C} \frac{e^{-x} x^{w-1}}{\Pi(x, n)} d x\right|<\frac{\Gamma((n-1) / 2)}{\Gamma(n / 2)} \frac{1+e^{v \pi / 2}}{4 \pi^{1 / 2}} \frac{e^{-c} c^{u-1}}{(a-c)^{n-1}}, \\
\left|\frac{1}{2 \pi i} \int_{C} \frac{x^{w-1}}{\Pi(x, n)} d x\right|<\frac{\Gamma((n-1) / 2)}{\Gamma(n / 2)} \frac{1+e^{v \pi / 2}}{4 \pi^{1 / 2}} \frac{c^{u-1}}{(a-c)^{n-1}},
\end{gathered}
$$

where $\Pi(x, n)=\Pi_{j=1}^{n}\left(x-x_{j}\right)$. 
Proof. The two estimates are much the same, so I only prove the first. Further, the estimates are empty if $n=1$, so assume $n \geqslant 2$. Denote the integral by $I$. Deform the contour to a vertical line segment with $\operatorname{Re}(x)=c$ and the arc of the circle $|x|=R$ to the right of $\operatorname{Re}(x)=c$. The integral on the arc of the circle is

Therefore

$$
\ll R R^{u-1} e^{v \pi / 2} / R^{n}=O\left(R^{1-n}\right) .
$$

$$
|I| \leqslant \frac{1}{2 \pi} \int_{-\infty}^{\infty} \frac{e^{-c}\left|(c+i t)^{w-1}\right|}{|a-(c+i t)|^{n}} d t
$$

since $a \leqslant x_{j}$ for $1 \leqslant j \leqslant n$. Then

$$
|I| \leqslant \frac{e^{-c}}{2 \pi}\left(1+e^{v \pi / 2}\right) \int_{0}^{\infty} \frac{\left(c^{2}+t^{2}\right)^{(u-1) / 2}}{\left((a-c)^{2}+t^{2}\right)^{n / 2}} d t
$$

which proves the estimate, since $u \leqslant 1$ and

$$
2 \int_{0}^{\infty}\left(1+z^{2}\right)^{-n / 2} d z=\frac{\Gamma(1 / 2) \Gamma((n-1) / 2)}{\Gamma(n / 2)} \text {. Q.E.D. }
$$

These estimates are useful in bounding the errors in the Gaussian integration formulae used below. An outline of the derivation of these formulae is presented so I can make use of the estimates just proved.

Let $\left\{p_{j}(x)\right\}$ be a family of monic polynomials with $\operatorname{deg}\left(p_{j}\right)=j$ and orthogonal with respect to the inner product defined by

$$
(f, g)=\int_{a}^{b} f(x) \overline{g(x)} w(x) d x
$$

where $w(x)$ is positive on $[a, b]$. Let $x_{1}, \cdots, x_{r}$ be the zeros of $p_{r}(x)$. An elementary result in the theory of orthogonal polynomials says that each $x_{j}$ is in $(a, b)$ and that the $x_{j}$ are distinct. Let $p(x)$ be the polynomial of degree $r-1$ satisfying $p\left(x_{j}\right)=g\left(x_{j}\right), j=1(1) r$, where $g(x)$ is some function analytic in a neighborhood of $[a, b]$, and let $q(x)$ be the polynomial of degree $r-1$ satisfying $\left(z-x_{j}\right)^{-1}=q\left(x_{j}\right), j=1(1) r$. The coefficients of $q(x)$ are rational functions of $z$. Then it is easy to verify that

$$
1 /(z-x)=q(x)+p_{r}(x) /(z-x) \Pi(z, r)
$$

and that

$$
\begin{aligned}
g(x) & =p(x)+\frac{p_{r}(x)}{2 \pi i} \int_{C} \frac{g(z)}{(z-x) \Pi(z, r)} d z \\
& =p(x)+\frac{p_{r}(x) q(x)}{2 \pi i} \int_{C} \frac{g(z)}{\Pi(z, r)} d z+\frac{p_{r}(x)^{2}}{2 \pi i} \int_{C} \frac{g(z)}{(z-x) \Pi(z, r)^{2}} d z .
\end{aligned}
$$

Then $\int_{a}^{b} p_{r}(x) q(x) w(x) d x=0$ since $\operatorname{deg}(q)<\operatorname{deg}\left(p_{r}\right)$, so 


$$
\int_{a}^{b} g(x) w(x) d x=\int_{a}^{b} p(x) w(x) d x+\int_{a}^{b} p_{r}(x)^{2} w(x) \frac{1}{2 \pi i} \int_{C} \frac{g(z)}{(z-x) \Pi(z, r)^{2}} d z d x
$$

Further, it is well known that

$$
\int_{a}^{b} p(x) w(x) d x=\sum_{j=1}^{r} a_{j} g\left(x_{j}\right)
$$

where the weights $a_{j}$ depend only on $w(x)$ and $r$. Thus, once the $a_{j}, x_{j}$ are known, (3) becomes an approximate integration formula in which the last term is the truncation error. For the classical orthogonal polynomials, $\int_{a}^{b} p_{r}(x)^{2} w(x) d x$ is known, so a bound for the contour integral in (3) provides a bound for the truncation error for the approximate integration formula. I only need two families of polynomials, the first orthogonal on $[-1,1]$ with $w(x)=1$, and the second orthogonal on $[0, \infty)$ with $w(x)=e^{-x}$. Except for constant factors, the first are the Legendre polynomials and the second are the Laguerre polynomials.

Lemma 4. Write $f(y)=e^{-y} y^{w-1}$ with $w=u+i v, 0 \leqslant u \leqslant 1, v \leqslant 0$. Let $x_{1}, \cdots, x_{r}$ be the zeros of the rth Legendre polynomial; let $b_{1}, \cdots, b_{r}$ be the corresponding weights in (4). Then

$$
\begin{aligned}
\mid \int_{a}^{b} f(y) d y- & \frac{b-a}{2} \sum_{j=1}^{r} b_{j} f\left(y_{j}\right) \mid \\
& <\frac{e^{-c} c^{u-1}}{(a-c)^{2 r}} \frac{1+e^{v \pi / 2}}{4 \pi^{1 / 2}} \frac{(r !)^{4}}{((2 r) !)^{2}} \frac{\Gamma(r)}{\Gamma(r+1 / 2)} \frac{(b-a)^{2 r+1}}{(2 r+1)},
\end{aligned}
$$

where $2 y_{j}=(b-a) x_{j}+a+b$.

Proof. Write $p_{r}(x)=\Pi(x, r)=\Pi_{j=1}^{r}\left(x-x_{j}\right)$. Then [6],

$$
\int_{-1}^{1} p_{r}(x)^{2} d x=\frac{2^{2 r+1}}{2 r+1} \frac{(r !)^{4}}{((2 r) !)^{2}}
$$

Let $x=(2 y-(a+b)) /(b-a)$, so that $\int_{a}^{b} f(y) d y=((b-a) / 2) \int_{-1}^{1} f(y) d x$. Now it follows from (3) that

$$
\left|\int_{-1}^{1} f(y) d x-\sum_{j=1}^{r} b_{j} f\left(y_{j}\right)\right|<\int_{-1}^{1} p_{r}(x)^{2}\left|\frac{1}{2 \pi i} \int_{D} \frac{f(((b-a) z+a+b) / 2)}{(z-x) \Pi(z, r)^{2}} d z\right| d x,
$$

where $D$ is a simple closed contour containing $x, x_{1}, \cdots, x_{r}$. Changing variables in the contour integral reveals that it is equal to

$$
(b-a)^{2 r} 2^{-2 r} \frac{1}{2 \pi i} \int_{C} \frac{f(t)}{(t-y) \Pi\left(t-y_{j}\right)^{2}} d t
$$

and an appeal to Lemma 3 completes the proof of this lemma. Q.E.D.

The proof of the following lemma is even simpler.

Lemma 5. Let $z_{1}, \cdots, z_{r}$ be the zeros of the rth Laguerre polynomial and 
let $c_{1}, \cdots, c_{r}$ be the corresponding weights in (4). Then

$$
\left|\int_{a}^{\infty} e^{-x} x^{w-1} d x-e^{-a} \sum_{j=1}^{r} c_{j}\left(z_{j}+a\right)^{w-1}\right|<(r !)^{2} \frac{\Gamma(r)}{\Gamma(r+1 / 2)} \frac{1+e^{v \pi / 2}}{4 \pi^{1 / 2}} \frac{c^{u-1} e^{-a}}{(a-c)^{2 r}} .
$$

In the last two lemmas $c$ is the free parameter of Lemma 3. The $x_{j}, z_{j}, b_{j}$, and $c_{j}$ are tabulated to 30 decimal places in [7]. When applying these lemmas one chooses $c$ so that the bound on the error is as small as possible.

The tools for evaluating $Z(t, \chi),|t| \leqslant 1 / 4$, are now at hand. In the calculation leading to the Table, for each $t$, the machine calculated a table of $\Gamma(3 / 4+i t / 2, \alpha)$, $\alpha=3.2(.2) 5.8(.4) 10.2(.8) 15.8$, by calculating $\Gamma(3 / 4+i t / 2,3.2)$ using Lemma 2 with $N=24$, and then calculating each successive function value from the previous value by 4-point Gaussian integration. (Lemma 4 with $r=4$.) Lemma 4 shows that none of the tabulated values has a truncation error of more than $10^{-12}$. As a measure of the actual error, the tabulated value of $\Gamma(3 / 4+i t / 2,15.8)$ minus a good approximation to $\int_{15.8}^{\infty} e^{-x} x^{s-1} d x$ was smaller, about $2 \cdot 10^{-15}$. The complicated form of the table was motivated by a desire for efficiency. One does not wish to spend too much time calculating the table, and since the values of $\alpha$ are not uniformly distributed, it seems reasonable to have fewer tabular values for large $\alpha$. The evaluation of any particular $\Gamma(3 / 4+i t / 2, \alpha)$ during the calculation of the series in Lemma 1 depends on the value of $\alpha$. If $\alpha<3.2$, the series of Lemma 2 is used, with $N$ chosen as indicated immediately after the proof of Lemma 2 . If $3.2 \leqslant \alpha \leqslant 16$, and $\alpha_{0}$ is the nearest argument for which the function value is tabulated, then

$$
\Gamma(.75+i t / 2, \alpha)=\Gamma\left(.75+i t / 2, \alpha_{0}\right)+\int_{\alpha_{0}}^{\alpha} e^{-x} x^{.75+i t / 2} d x / x,
$$

and the last integral is approximated by 3-point Gauss-Legendre integration, and the truncation error is bounded using Lemma 4. Finally, if $\alpha>16$, the value of $\Gamma(.75+i t / 2, \alpha)$ is approximated by 4-point Gauss-Laguerre integration as in Lemma 5. Using Lemmas 4 and 5 it is easy to see that the total truncation error in any incomplete $\Gamma$-function is no more than about $10^{-12}$, so that this error contributes no more than $3 \cdot 10^{-12} k$ to the error in the series of Lemma 1 . Thus, for $k$ around $10^{5}$, the truncation error from the incomplete $\Gamma$-functions appears to be the dominant source of error in the calculated values of $Z(t, \chi)$.

In addition to the errors already considered, there are inaccuracies in the calculation of $e^{x}, \log x$, and other elementary functions, and errors due to finite-precision arithmetic, all usually no more than one part in $10^{15}$. One can estimate the effect of these sources of error on the accuracy of the calculated value of $Z(t, \chi)$. Also it is necessary to check the program very carefully to make sure it is doing what it is supposed to. In place of these otherwise necessary and probably unconvincing details, the next section contains another, similar way of calculating the zeros of the Table, which gives the same answers, but which uses none of the calculations made by the program discussed above. 
3. Another Function. If $\chi(-1)=-1$, then $\zeta(s) L(s, \chi)=\zeta_{K}(s)$, the zeta function of the complex quadratic field $K=Q(\sqrt{-k}), k$ being the conductor of the real valued character $\chi$. The functional equation for $\zeta_{K}(s)$ can be written in a form similar to that of (1), namely

$$
\begin{aligned}
\xi_{K}(s)= & \left(\frac{k^{1 / 2}}{2 \pi}\right)^{s} \Gamma(s) \zeta_{K}(s)=\frac{h(K)}{2 s(s-1)}+\left(\frac{k^{1 / 2}}{2 \pi}\right)^{s} \sum_{n=1}^{\infty} \frac{r(n)}{n^{s}} \Gamma\left(s, 2 \pi n / k^{1 / 2}\right) \\
& +\left(\frac{k^{1 / 2}}{2 \pi}\right)^{1-s} \sum_{n=1}^{\infty} \frac{r(n)}{n^{1-s}} \Gamma\left(1-s, 2 \pi n / k^{1 / 2}\right)
\end{aligned}
$$

where $k>4, h(K)$ is the ideal class number of $K$, and $r(n)=\Sigma_{j \mid n} \chi(j)$.

If $s=1 / 2+i t$, then the function $Z_{K}(t)=\xi_{K}(.5+i t)$ is real and has its zeros at exactly those $t$ at which $\zeta(s) L(s, \chi)$ is 0 . Since the smallest zero of $\zeta(s)$ has $t>14$, any zeros of $Z_{K}(t)$ with $0 \leqslant t \leqslant 14$ must be zeros of $L(s, \chi)$. Hence formula (5) can be used in place of formula (1) for finding zeros of $L(s, \chi)$. A potential advantage to using (5) is that many of the $r(n)$ will be zero, so that calculating $\xi_{K}(s)$ may require calculating fewer terms in the infinite series. Formula (5) also requires the value of $h(K)$.

Lemma 6. If $s=1 / 2+i t$ and $k>4$, then

$$
\left|Z_{K}(t)-2 \operatorname{Re}\left[\frac{h(K)}{s(s-1)}+\left(\frac{k^{1 / 2}}{2 \pi}\right)^{s} \sum_{n=1}^{\infty} \frac{r(n)}{n^{s}} \Gamma\left(s, 2 \pi n / k^{1 / 2}\right)\right]\right|<\frac{k e^{-2 \pi N / k^{1 / 2}}}{\pi^{2} N^{3 / 2}} .
$$

Proof. The left-hand side is bounded by

$$
2\left(\frac{k^{1 / 2}}{2 \pi}\right)^{1 / 2} \sum_{N+1}^{\infty} \frac{r(n)}{n^{1 / 2}} \Gamma\left(.5,2 \pi n / k^{1 / 2}\right) \leqslant 2\left(\frac{k^{1 / 2}}{2}\right)^{1 / 2} \int_{N}^{\infty} 2 \int_{2 \pi u / \sqrt{k}}^{\infty} x^{-1 / 2} e^{-x} d x d u
$$

since $r(n) \leqslant d(n) \leqslant 2 n^{1 / 2}$. This last bound is no larger than

$$
\frac{2 \sqrt{k}}{\pi} \int_{N}^{\infty} \frac{e^{-2 \pi u / k^{1 / 2}}}{\sqrt{u}} d u \leqslant \frac{k}{\pi^{2} N^{3 / 2}} e^{-2 \pi N / \sqrt{k}} \text {. Q.E.D. }
$$

The truncation error in Lemma 6 is somewhat larger than the error term in Lemma 1, at least for the $k$ used in this paper. The program used to calculate $Z_{K}(t)$ works in much the same way that the program for $Z(t, \chi)$ does. For each $t$ this program tabulates $\Gamma(.5+i t, \alpha)$ for $\alpha=3(.5) 13$ using 6-point Gauss-Legendre integration. The program uses the series of Lemma 2 if $\alpha<3$, and 6-point Gauss-Laguerre integration if $\alpha>13$, while for intermediate values of $\alpha$ it interpolates in the Table using 6-point Gauss-Legendre integration. A discussion of the accuracy with which $Z_{K}(t)$ can be calculated is available in [2]. The bounds given there depend on Lemmas 4 and 5 , and are very conservative. 


\begin{tabular}{rccccc}
\multicolumn{7}{c}{ TA B LE } \\
\hline \multicolumn{1}{r}{$k$} & $\gamma$ & $Z_{K}(\gamma)^{\prime}$ & $Z(\gamma, \chi)^{\prime}$ & $\frac{\gamma \log k}{2 \pi}$ & $\begin{array}{c}\text { guaranteed } \\
\text { accuracy } \\
\times 10^{10}\end{array}$ \\
\hline 163 & .202901338 & 2.1 & -15.7 & .164 & 5 \\
427 & .249924977 & 4.1 & -54 & .241 & 5 \\
2683 & .156678803 & 7.3 & -210 & .197 & 5 \\
17923 & .030985799 & 4.6 & -310 & .0483 & 8 \\
28963 & .033774095 & 4.7 & -400 & .0552 & 10 \\
30895 & .018493558 & 10.3 & -910 & .0304 & 6 \\
37427 & .019504713 & 7.6 & -740 & .0327 & 7 \\
115147 & .003157614 & .73 & -120 & .00586 & 95 \\
123204 & .010649913 & 9.2 & -1620 & .0199 & 10 \\
139011 & .012930178 & 9.4 & -1760 & .0244 & 10 \\
145412 & .017311754 & 13.6 & -2600 & .0328 & 10 \\
151419 & .021346721 & 12.5 & -2450 & .0405 & 10 \\
188995 & .026513007 & 9.0 & -1970 & .0513 & 12 \\
991027 & .151734485 & 2.4 & -1320 & .333 & 75 \\
\hline
\end{tabular}

4. Calculating the Zeros. In this section I use $Z(t)$ to denote either $Z(t, \chi)$ or $Z_{K}(t)$. To find a zero of $Z(t)$ the program locates an interval in which $Z(t)$ changes sign, and then uses the secant method to get to a point where the calculated value of $Z(t)$ is very small. In every calculation the secant method converged very rapidly. The point to which the secant method converges is taken to be an approximation to a zero of $Z(t)$. These are the values given in the column headed $\gamma$ in the Table. The next two columns in the Table are calculated values of $Z^{\prime}(\gamma)$. If the values of $Z(t)$ are known to within an error of $\epsilon$ and if the value of $Z^{\prime}$ is known, then the true zero cannot differ from the calculated zero by more than $\epsilon /\left|Z^{\prime}\right|$. This observation is the source of the column labelled "guaranteed accuracy $\times 10^{10}$ ". The calculated values of $\gamma$ are all given to nine decimal places even when the two programs did not give such an accurate answer. In these cases the value given is an average of the values calculated. The column headed $(\gamma \log k) / 2 \pi$ gives a measure of how close the zero is to the real axis.

Except for $k=991027$, the values of $k$ in the Table are just those from [2]. In [2] some of the $\gamma$ were necessary to prove a theorem about small class numbers. The proof would go through if the values were only known with an accuracy of one part in 20, so for this purpose the accuracy of the Table is not needed. The value $k=991027$ is included because $h(K)=63$, according to Daniel Shanks, which is very small compared to the size of $k$. This example shows that closeness of $\gamma$ to 0 is not invariably implied by small class number. 
5. Other Characters. It is clear that (1) and the results of Section 2 can be used without change when $\chi$ is real-valued and $\chi(-1)=1$. Unfortunately, the formula corresponding to $(5)$ for these characters is much less convenient, since it contains double integrals in place of the integral defining $\Gamma(s, \alpha)$. It does not seem to be possible to use this formula to find zeros of $L(s, \chi)$, so there is no independent check available for zeros calculated using (1).

The generalization of (1) to any primitive character is

$$
\xi(s, \chi)=\left(\frac{k}{\pi}\right)^{(s+a) / 2} \Gamma\left(\frac{s+a}{2}\right) L(s, \chi)=\left(\frac{k}{\pi}\right)^{(s+a) / 2} \sum_{n=1}^{\infty} \frac{\chi(n)}{n^{s}} \Gamma\left(\frac{s+a}{2}, \frac{\pi n^{2}}{k}\right)
$$

$$
+\frac{i^{a} k^{1 / 2}}{\tau(\bar{\chi})}\left(\frac{k}{\pi}\right)^{(1-s+a) / 2} \sum_{n=1}^{\infty} \frac{\chi(n)}{n^{1-s}} \Gamma\left(\frac{1-s+a}{2}, \frac{\pi n^{2}}{k}\right),
$$

where $\tau(\chi)=\sum_{j=1}^{k} \chi(j) e^{2 \pi i j / k}$.

The same arguments that prove Lemmas $1,4,5$ show that $O\left(k^{1 / 2} \log k\right)$ terms of the series give $\xi(s, \chi)$ accurately, if $|t|$ is small, but it appears that the evaluation of $\tau(\chi)$ requires nearly $k$ computations. However, H. Montgomery observed that the functional equation for theta functions used in the proof of (6) contains $\tau(\bar{\chi})$. Write

$$
\theta(x, \chi)=\sum_{n=1}^{\infty} \chi(n) e^{-\pi n^{2} x / k}, \quad \theta_{1}(x, \chi)=\sum_{n=1}^{\infty} n \chi(n) e^{-\pi n^{2} x / k} .
$$

Then $\left[8\right.$, p. 70] if $\chi(-1)=1$, it is true that $\tau(\bar{\chi}) \theta(x, \chi)=(k / x)^{1 / 2} \theta(1 / x, \bar{\chi})$, and if $\chi(-1)=-1, \tau(\bar{\chi}) \theta_{1}(x, \chi)=i k^{1 / 2} x^{-3 / 2} \theta_{1}(1 / x, \bar{\chi})$. Taking $x=1$ gives

$$
\begin{aligned}
& \tau(\bar{\chi})=k^{1 / 2} \exp \left(i \text { arg } \sum_{n=1}^{\infty} \bar{\chi}(n) e^{-\pi n^{2} / k}\right), \quad \text { if } \chi(-1)=1, \\
& \tau(\bar{\chi})=k^{1 / 2} \exp \left(i \arg \sum_{n=1}^{\infty} n \bar{\chi}(n) e^{-\pi n^{2} / k}\right), \quad \text { if } \chi(-1)=-1 .
\end{aligned}
$$

Now

$$
\left|\sum_{N+1}^{\infty} n \bar{\chi}(n) e^{-\pi n^{2} / k}\right| \leqslant \int_{N}^{\infty} x e^{-\pi x^{2} / k} d x=\frac{k}{2 \pi} e^{-\pi N^{2} / k}
$$

therefore $O\left(k^{1 / 2} \log k\right)$ terms of the appropriate series are enough to calculate the Gauss sum.

Department of Mathematics

University of Michigan

Ann Arbor, Michigan 48104

1. J. B. ROSSER, J. M. YOHE \& L. SCHOENFELD, "Rigorous computation and the zeros of the Riemann zeta-function," Proc. IFIP Congress (Edinburgh, 1968), vol. 1: Mathematics, Software, North-Holland, Amsterdam, 1969, pp. 70-76. MR 41 \#2892. 
2. H. L. MONTGOMERY \& P. J. WEINBERGER, "Notes on small class numbers," Acta Arith., v. 24, 1974, pp. 529-542.

3. D. DAVIES \& C. B. HASELGROVE, "The evaluation of Dirichlet $L$-functions," Proc. Roy. Soc. Ser. A, v. 264, 1961, pp. 122-132. MR 24 \#B2091.

4. D. DAVIES, “An approximate functional equation for Dirichlet $L$-functions," Proc. Roy. Soc. Ser. $A$, v. 284,1965 , pp. 224-236. MR 30 \#356.

5. D. H. LEHMER, "Extended computation of the Riemann zeta-function," Mathematika, v. 3, 1956, pp. 102-108. MR 19, $121 ; 1431$.

6. M. ABRAMOWITZ \& I. A. STEGUN (Editors), Handbook of Mathematical Functions, With Formulas, Graphs and Mathematical Tables, 3rd printing with corrections, Nat. Bur. Standards Appl. Math. Series, 55, Superintendent of Documents, U. S. Government Printing Office, Washington, D. C., 1965. MR 31 \#1400.

7. A. H. STROUD \& D. SECREST, Gaussian Quadrature Formulas, Prentice-Hall, Englewood Cliffs, N. J., 1966. MR 34 \#2185.

8. H. DAVENPORT, Multiplicative Number Theory, Lectures in Advanced Math., no. 1, Markham, Chicago, Ill., 1967. MR 36 \#117.

9. M. E. LOW, "Real zeros of the Dedekind zeta function of an imaginary quadratic field," Acta. Arith., v. 14, 1967/68, pp. 117-140. MR 38 \#425.

10. G. PURDY, "The real zeros of the Epstein zeta function.” (To appear). 